\title{
THE EVALUATION OF THE INITIAL SKEW RATE FOR PRINTED TEXT
}

\author{
Darko Brodić
}

\begin{abstract}
In this manuscript the algorithm for identification of the initial skew rate for printed text is presented. Proposed algorithm creates rectangular hull around all text characters. Combining nearby rectangular hulls form objects. After applying mathematical morphology on it, the biggest object is characterized as well as selected. Rectangular hull gravity center forms reference points on these objects used as a base for calculation ieestimation of the initial skew rate. Using the least square method, initial skew rate is calculated. Comparative analysis of the origin and estimated skew rate is presented as well as discussed. Algorithm is examined with a number of printed text examples. Proposed algorithm showed robustness for skewness of printed text in the wide range.

K e y w ord s: OCR, document image processing, skew rate, printed text
\end{abstract}

\section{INTRODUCTION}

Nowadays, most of the information is saved in electronic form. However, old documentation is primarily in the printed form. Converting documentation is tedious job. Mainly, it is done by scanners. They convert documentation from paper to electronic form. The main stage in this process is transformation of digital document image into ASCII characters. Optical character recognition (OCR) is a system responsible for that role. OCR works fine if the scanned document is not skewed. However, to achieve its complete accuracy document should be skewness and noiseless. Hence, the skew identification is the main role in the processing stage prior to the stage of the character recognition in OCR [1].

As stated previosly, old text documentation is completely built from printed text. Printed text is defined by its characteristics. Firstly, it is well-formed text type. Hence, it has strong regularity in shape. Consequently, if different text lines have any orientation then it will be very similar. Therefore, they have so-called uniform skewness. Further, their orientations are not significantly variable on same page of the text. Also, descendant and ascendant characters from neighbor text lines have decent spacing. So, they are not linked with each other's. Distances between the lines have enough space to split it up. Besides, words in text lines are formed regularly with similar distance and their inter word spacing is decent as well. Hence, printed text from the documentation is in some way predictable one. Existing skew identification techniques can be classified in the following directions [1]:

- Histogram analysis,

- K-nearest neighbor clustering,

- Projection profile,

- Fourier transform,
- Cross-correlation,

- Other models.

In [2] it is proposed the technique of reference line extraction based on identifying valleys of horizontal pixel density histogram. Method is suited for uniform skew rate but failed for multi-skewed text lines, and it is time consuming.

The Docstrum as K-nearest neighbor clustering method [3] is by product of a larger page layout analysis system, which assumed that only text is being processed. The connected components formed by the nearest neighbors clustering are essentially characters only. The method is suitable for finding skew angle. But, it is limited to Roman languages due to poor text line segmentation.

Another method proposed in [4] deal with simple multi-skewed text. It uses as a basis simple type of Hough transform for straight lines. But, it is too specific and computer intensive.

The Fourier transform method is a representation in the Fourier domain of the projection profile method in the pixel domain. The results are mathematically identical, but Fourier transform is only different approach to the same text and document properties projection profile is based upon [5].

The cross-correlation method calculates both horizontal and vertical projection profiles and then compares the shift inter-line cross-correlation to determine the skew rate. Although method can handle complex layout structure documents, applied range is limited to small skew rate angles up to $10^{\circ}[6]$.

Method of identifying words contour area as a start for referent line detection is proposed in [7]. Still, the assumptions made on the definition of word elements are too specific.

\footnotetext{
* University of Belgrade, Technical Faculty Bor, V. J. 12, 19210 Bor, Serbia, dbrodic@tf.bor.ac.rs
} 


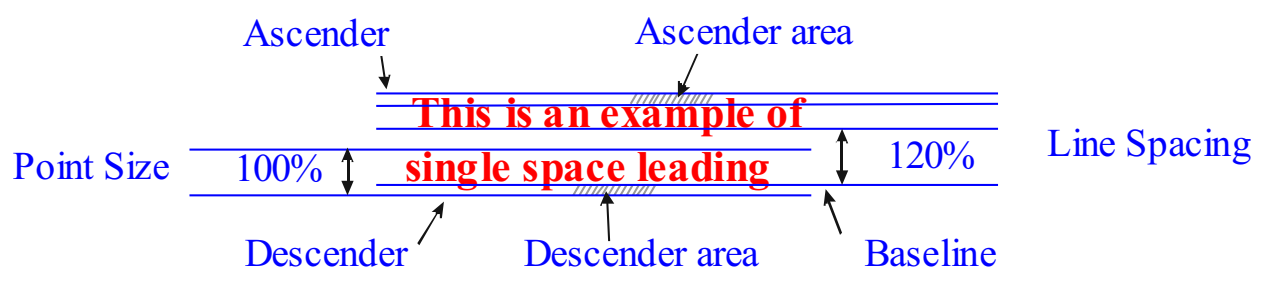

Fig. 1. Definition of the typical printed text leading

"Water flow" method [5] assumed hypothetical flow of water in a particular direction across image frame in a way that it faces obstruction from the characters of the text lines. This method is adopted in [8]. Algorithm is robust, but in specific circumstance is unpredictable. Hence, it needs further improvements.

Simple method based on skew detection on the polygon centre of gravity is proposed in [9]. Simplicity and global approach is an advantage of it. However, accuracy is $\mathrm{u}$ to $87 \%$ [9] making its use questionable under different condition and circumstance.

Another simple method based on boundary growing approach exclusively for printed text is proposed in [10]. Although simple method, making its refinement caused it to be time consuming. Still, it has some constraints. In addition, some pre assumptions are needed to be successful.

Modification of the method [10] is presented in [11]. Proposed method advanced original method and solve some deficiencies. Using text image dilatation, algorithm extended its usability and accuracy. Comparing with other methods, obtained results are promising. Still, upgraded method is computer time consuming. Main characteristics of the skew detection techniques are algorithm accuracy and its computational complexity. Techniques classified as other models mostly represent morphological and geometrical transformation algorithms or its derivations. Those algorithm are computational inexpensive. Hence, those methods are in the scope of the paper. Hence, in this paper modification of the method based on the rectangular hull and its gravity center points [10] is presented. Initial method is extended by simplifying it. Original paper constraints are overcome making upgraded algorithm more robust and applicable. Organization of this paper is as follows. Section 2 includes brief description and information on proposed algorithm based on rectangular hull gravity center. In Section 3 text experiment is defined. In Section 4 obtained results are examined and discussed. In Section 5 conclusion is made as well as further investigation direction is pointed out.

\section{THEORETHICAL PART}

At the beginning of the test process, original image is used. Document text image is obtained as product of original image scanning. Document text image is digital text image represented by matrix $\mathbf{D}$ with $M$ rows, $N$ columns, and intensity with $L$ discrete levels of gray. $L$ is the integer number from the set $\{0, \ldots, 255\}$. Currently, $D(i, j) \in\{0, \ldots, 255\}$, where $i=1, \ldots, M$ and $j=1, \ldots, N$. After applying intensity segmentation with binarization, intensity function is converted into binary intensity function given by

$$
B(i, j)= \begin{cases}1, & D(i, j) \geq D_{\text {th }} \\ 0, & D(i, j)<D_{\text {th }}\end{cases}
$$

where $D_{\text {th }}$ is given by Otsu algorithm [12] or equivalent algorithm [13-15]. It represents threshold sensitivity decision value. Currently, document image is represented as binary matrix B featuring $M$ rows by $N$ columns. Consequently, it consists of the only black and white pixels where value 0 represents black pixels and value 1 white pixels.

Further, binary document image should be "prepared" for text feature extraction by decomposition of document image to text and non-text regions. It is assumed that only text area is extracted. In addition, some morphological preprocessing is performed to make document image "noiseless". The morphological procedure was defined in [16] by the morphological closing of the opening the document binary image

$$
\mathbf{X}=(\mathbf{B} \circ \mathbf{S}) \bullet S
$$

For morphological operation, simple structuring element $\mathbf{S}$ dimension $3 \times 3$ with only ones is used [16]. This way, the morphological operation eliminates noise made by isolated black pixels in the image. Original algorithm main constraint [10] was assumption that text line spacing is always sufficient. Thus, boundary-growing region made by algorithm that extends text areas will not connect nearby text lines. Algorithm makes rectangular hull around every character or text object. To distinct every text line, each rectangular hull is growing in all directions to the nearby rectangular hull of the next character or text object. This process facilitates text line segmentation, but may lead to joining and merging nearby text lines. Hence, in real situation, above assumption is ambiguous.

Definition of the typical printed text leading $i e$ line spacing is given in Fig. 1. 

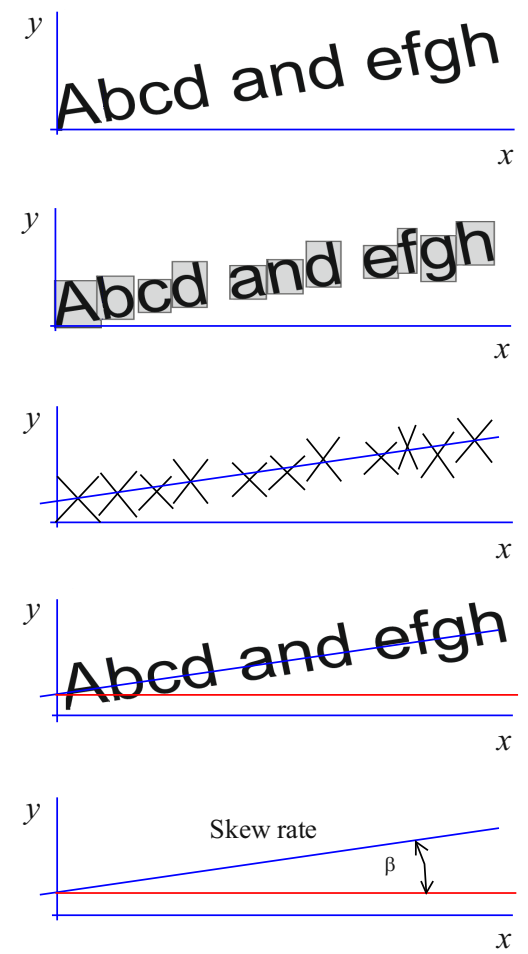

Fig. 2. Initial skew rate estimation process: (a) - Initial text, (b) - Rectangular hull around each character, (c) - Rectangular hull objects, (d) - Rectangular hull gravity center points, (e) - Initial skew rate estimation, (f) - Initial skew rate identification.

The first step of the algorithm is the extraction of the bounding box over the text objects. This is the prerequisite. A finite number of linear inequalities specify interior of the bounding box. Special case of the bounding box is a rectangular region whose edges are parallel to their coordinate axes. It is defined by its maximum and minimum extents for all axes. Hence, each pixel $X(i, j)$ belongs to the box is given by [17]

$$
X(i, j) \mid\left(x_{\min } \leq i \leq x_{\max }\right) \wedge\left(y_{\min } \leq j \leq y_{\max }\right) .
$$

Consequently, bounding box is defined by its endpoints $x_{\min }, y_{\min }, x_{\max }, y_{\max }$. Inclusion of the point $X(i, j)$ in a box is tested by verifying these four inequalities in (3). If any one of them fails, then the point is not inside. Rectangular hull growing around character by $10 \%$ [18] in each direction may lead to merging nearby text lines. Still, making justified text alignment led to extended text space barrier between words. Hence, algorithm ought to extend growing area joining rectangular hull objects. In our method, boundary-growing approach is based on rectangular hull gravity center as well. Steps in Fig. 2(a)-(f) give boundary growing and its text gravity center points.
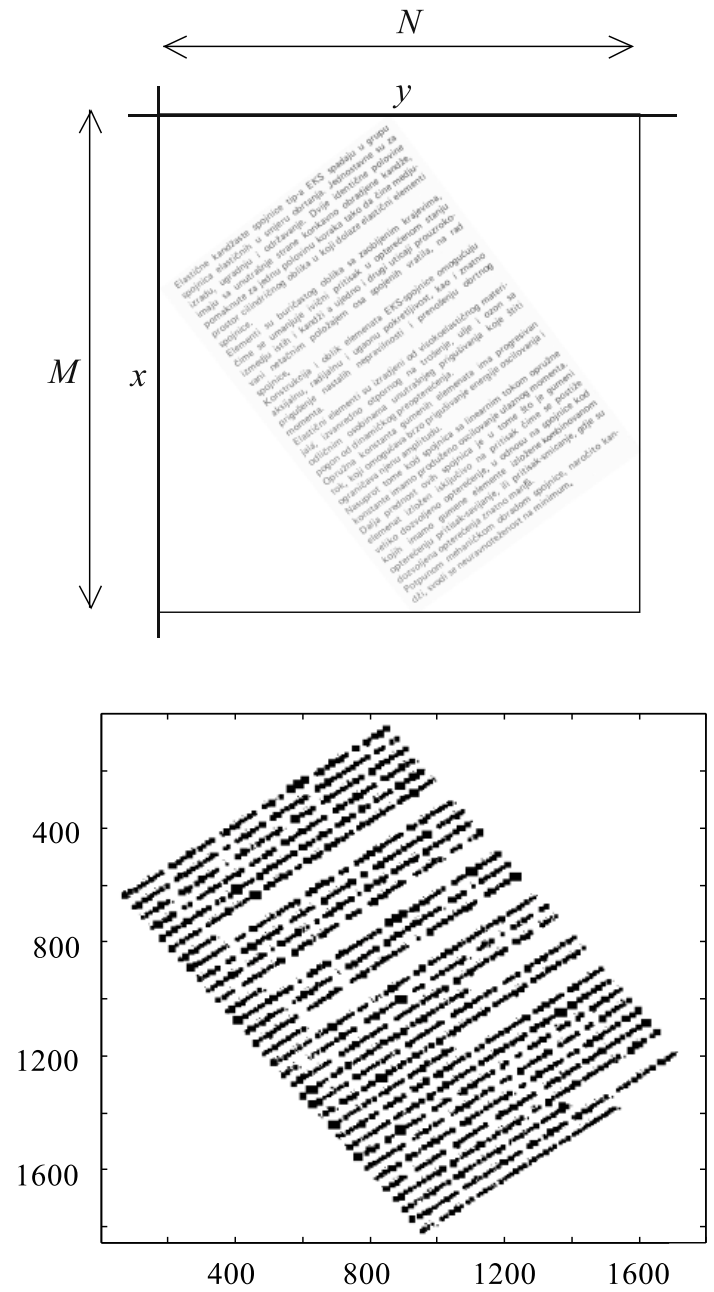

Fig. 3. Document binary image and its counterpart with rectangular hull objects: (a) - Matrix D, (b) - Matrix $\mathbf{Y}$

All characters or part of word is surrounded by rectangular hull, thus making boundary-growing area of the new matrix $\mathbf{Y}$. Scanned document text image as well as its counterpart with rectangular hulls object is given in Fig. 3.

Every rectangular hull around text is represented by its gravity center. Each rectangular object gravity center is given by pixel. Those pixels are corner stone for referent text line calculation ie estimation. Further, obtained referent text line is a prerequisite for skew rate identification.

Main difference between original algorithm [10] and our approach are in text segmentation approach. In our case, current rectangular hull is not growing in all directions to the nearby rectangular hull of the next character. Thus, main assumption on sufficient text line spacing is unnecessary. Characters close to each other make rectangular hulls mutually connected forming longer object containing set of different gravity center points.

The longest few objects $O_{l}$ extracted by morphological erosion from the set of objects $O_{\mathrm{ALL}}$ are used. Consequently, erosion structuring element $\mathbf{S}_{E}^{n}$ defined as variable width line is used. 


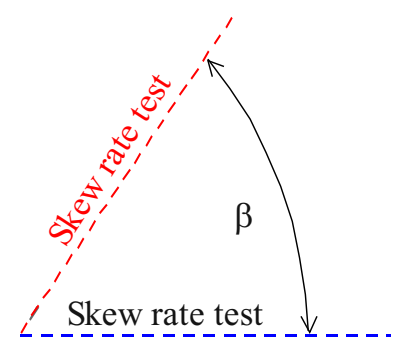

Fig. 4. Printed text experiment

Erosion is given by

$$
\mathbf{Z}^{n}=\mathbf{Y} \Theta \mathbf{S}_{E}^{n},
$$

where $n=1, \ldots, U$, and $l=1, \ldots, V$. It is assumed $U=3$ and $V=5$. It means three variable width lines defined by $n$ and the longest five objects defined by $l$ are used. From the experiment, results show that extending the number of structuring elements beyond defined number of $n$ represented by $U=3$ is unnecessary. This way, it is sufficient for extracting initial skew rate. In addition, extending the number of the longest objects is computationally expensive and results are not better. Finally, the longest object $O_{\mathrm{LNG}}$ is obtained by the following relation

$$
Z(i, j)=\bigcap_{n=1}^{U} Z^{n}(i, j)
$$

Longest objects from $\mathbf{Z}$ can be found out by so-called longest common subsequence (LCS) pseudo-code

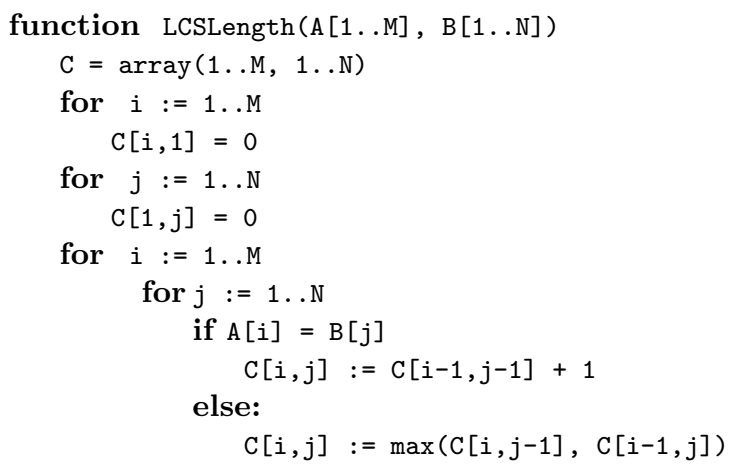

return $\mathrm{C}[\mathrm{m}, \mathrm{n}]$

where matrix $\mathbf{Z}$ is defined by vectors $\mathbf{A}$ and $\mathbf{B}$ and resulted object is defined by matrix $\mathbf{C}$.

Further, each object $O_{l}$ is represented by the set of rectangular hulls as its integral part. Similarly, each of those rectangular hulls characterized its gravity center points. Gravity center pixels defined by its rectangular hull maximum and minimum of $x$ and $y$ coordinates $i e$ $x_{\max }, x_{\min }, y_{\max }, y_{\min }$, respectively. Definition of the gravity center pixel is given by

$$
G C_{K, l}(x, y)=\left(\frac{x_{\min }+x_{\max }}{2}, \frac{y_{\min }+y_{\max }}{2}\right) .
$$

Furthermore, array of $G C_{K, l}(x, y)$ is defined for $K=$ $1, \ldots, R_{l}$ where $R_{l}$ represents the number of rectangular hulls of the longest objects $O_{l}$ and as stated $l=5$. Gravity center definition in (6) is general, but in our case can be applied for gravity center points adhered to rectangular hulls forming the longest five objects $O_{l}$. Due to the linear aspect of the referent text line, its restoring can be made by some numerical method. To solve this problem, least square method is used. Firstdegree polynomial function approximation is given by

$$
y=a_{l}^{\prime} x+b_{l}^{\prime} .
$$

where $m_{l}$ is the number of data points of the array $G C_{K, l}$, while slope $a_{l}^{\prime}$, and $y$-intercept $b_{l}^{\prime}$ are calculated as $[19]$

$$
a_{l}^{\prime}=\frac{\left(\sum y_{K, l}\right) *\left(\sum x_{K, l} y_{K, l}\right)-m_{L} *\left(\sum x_{K, l} y_{K, l}\right)}{\left(\sum x_{K, l}\right)^{2}-m_{l}\left(\sum x_{K, l}^{2}\right)},
$$

and

$$
b_{l}^{\prime}=\frac{\left(\sum x_{K, l}\right) *\left(\sum x_{K, l} y_{K, l}\right)-\left(\sum y_{K, l}\right) *\left(\sum x_{K, l}^{2}\right)}{\left(\sum x_{K, l}\right)^{2}-m_{l} *\left(\sum x_{K, l}^{2}\right)}
$$

After completing calculation by using (8) and (9), reference text line defined by $(7)$ is estimated. From obtained reference text line, skew rate is calculated ieestimated. For each object $l$, calculated referent text line has been represented by group of pixels $G C_{K, l}\left(x_{K, l}, y_{K, l}\right)$. Initial skew rate for each object is defined as

$$
\beta_{l}=\arctan \left(a_{l}\right)
$$

Still, each object skew rate is similar, but different. Estimated initial skew rate obtained by averaging the skew rate set of the longest objects are [18]

$$
\beta_{E S T}=\frac{\sum_{l=1}^{V} \beta_{l}}{V} .
$$

After obtaining initial skew rate, document text image is rotating for that angle. Real skew rate is close to the initial skew rate. However, it means a final adjustment is needed. It can be made on rotated text image by repeating above method ie iterating it.

Similarly, to accomplish text line segmentation other methods can be applied. After finishing segmentation by using horizontal and vertical projection profile, all text lines are disconnected. Now, proposed method on single text line can be applied. However, this process of the skew rate final adjustment is out of scope of the paper.

\section{EXPERIMENTAL PART}

For the experiment, printed sample text rotated up to $90^{\circ}$ by step of $5^{\circ}$ to $10^{\circ}$ around $x$-axis is used. Example of printed sample text is given in Fig. 4. 
Table 1. $R L H R$ of the rectangular hull gravity center (RHGC) algorithm

\begin{tabular}{|c|c|c|c|c|c|c|c|c|c|c|c|c|c|}
\hline Text $A$ & $5^{\circ}$ & $10^{\circ}$ & $15^{\circ}$ & $20^{\circ}$ & $25^{\circ}$ & $30^{\circ}$ & $35^{\circ}$ & $40^{\circ}$ & $45^{\circ}$ & $50^{\circ}$ & $60^{\circ}$ & $70^{\circ}$ & $80^{\circ}$ \\
\hline$\beta_{\mathrm{REF}}$ & 0.087 & 0.1763 & 0.2679 & 0.3639 & 0.4663 & 0.5773 & 0.7002 & 0.8391 & 1.0000 & 1.1917 & 1.732 & 2.7474 & 5.6713 \\
\hline$\beta_{\mathrm{EST}}$ & 0.0879 & 0.17 & 0. & 0. & 0.4 & 0.5856 & 0.7 & 0.8514 & 1.0119 & 1.2038 & 1.7488 & 42 & 5.7169 \\
\hline$R E=(\beta) \Delta \beta / \beta$ & 0.0 & 0 & 0. & 0. & 0 . & 0 & 0 & 0.1 & ( & 0. & 0.0091 & 0.0001 & 0.0080 \\
\hline$R L H R(\%)$ & 100.57 & 101. & 100.9 & 101.21 & 101.29 & 101.44 & 101.77 & 101.47 & 101.19 & 101.02 & 100.97 & 100.61 & 100.80 \\
\hline$R M S E$ & 0.0005 & 0.001 & 0.001 & 0.0027 & 0.0036 & 0.0047 & 0.0064 & 0.0074 & 0.0080 & 0.0085 & 0.0096 & 0.010 & 0.0161 \\
\hline
\end{tabular}

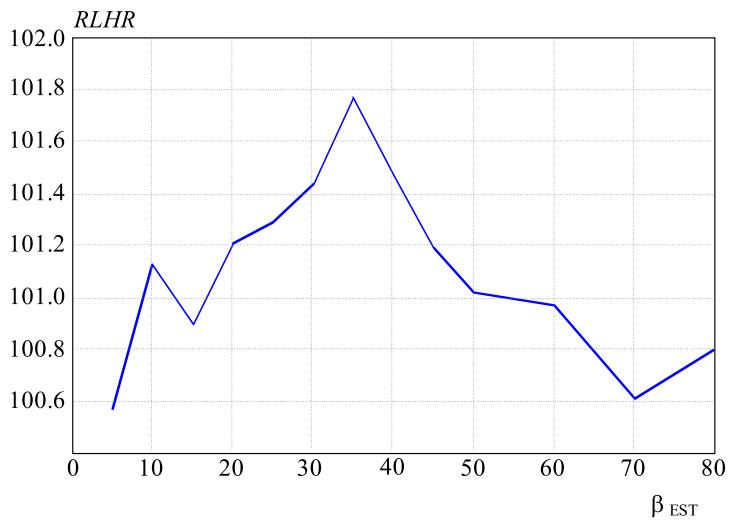

Fig. 5. $R L H R$ of the rectangular hull gravity center (RHGC) algorithm without erosion

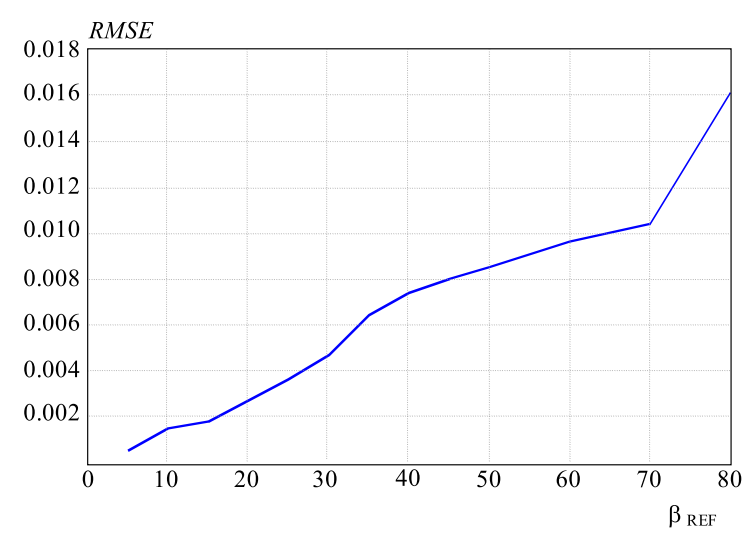

Fig. 6. RMSE of the rectangular hull gravity center (RHGC) algorithm without erosion

This sample text reference line is represented by

$$
y=a x+b .
$$

Furthermore, referent line hit rate ie $R L H R$ represents the algorithm succeed measure of retracing original referent text line defined by [20]

$$
R L H R=1-R E(\beta)=1-\frac{\Delta \beta}{\beta}=1-\frac{\beta_{R E F}-\beta_{E S T}}{\beta_{R E F}} .
$$

where $R E(\beta)$ represents relative error from $\beta$, while $\beta_{R E F}$ is arctan of $a$ given in (12) as well as $\beta_{E S T}$ is arctan of $a_{l}^{\prime}$ given in (7).

Root mean square ( $R M S E)$ value is calculated by [20]

$$
R M S E=\sqrt{\frac{1}{R} \sum_{k=1}^{R}\left(x_{R E F}^{k}-x_{E S T}^{k}\right)^{2}} .
$$

where $k=1, \ldots, R$. Furher, $R$ is the number of examined text rotating angles up to $90^{\circ}, x_{R E F}$ is $R L H R$ for $\beta_{E S T}$ equal to $\beta_{R E F}$ ie due to normalization equal to 1 , and $x_{E S T}$ is $R L H R$.

\section{DISCUSSION}

After applying proposed algorithm to the printed sample text in the whole region $\left\{0^{\circ}, \ldots, 90^{\circ}\right\}$ obtained $R L H R$ is presented in Tab. 1. In this case, proposed algorithm without morphological erosion in (4) is applied. But, it is applied only to the single text line. This experiment is valid for examination of the proposed algorithm quality, accuracy and usability. Data from Tab. 1. are shown in Fig. 5.

$R L H R$ for printed sample text, in examined rotation angle region $i e$ whole region of $\left\{0^{\circ}, \ldots, 90^{\circ}\right\}$, is very good. In other words, examined algorithm retraces original referent text line almost completely accurate. Evidence of that is given by $R M S E$ figure. It is shown in Fig. 6.

Especially, RMSE figure is almost linear function of rotation angle up to $70^{\circ}$. This is very rare in the domain of skew detection algorithms. Still, good retracing figure ie small scattering of the results contribute to a small $R M S E$ value of algorithm.

Further, proposed algorithm is applied on multi line text. This experiment is used for initial skew rate extraction. In this experiment, proposed algorithm is extended by morphological erosion in (4). Parameter linked with image erosion represents the remaining number of the objects $O_{\mathrm{ALL}}$, after applying it. However, after each erosion iteration enforcement, the number of the remaining image objects is decreasing. From remained number of still existing objects, only the longest five is chosen for initial skew rate estimation. For experiment, printed text samples were multi line text rotated in the region $\left\{0^{\circ}, \ldots, 90^{\circ}\right\}$ by step $10^{\circ}$. Obtained results are presented in Tab. 2 and 3. These tables represent results that depend from parameter of the remained object number $O_{\mathrm{ALL}}$. Data from Tab. 2 and 3 are given in Fig. 7 and Fig. 8. 
Table 2. Initial $R L H R$ of the RHGC algorithm extended by erosion with step $O_{\mathrm{ALL}} \leq 50$ objects

\begin{tabular}{ccccccccccc}
\hline Text Angle & $5^{\circ}$ & $10^{\circ}$ & $20^{\circ}$ & $30^{\circ}$ & $40^{\circ}$ & $50^{\circ}$ & $60^{\circ}$ & $70^{\circ}$ & $80^{\circ}$ & $85^{\circ}$ \\
\hline$\beta_{\mathrm{REF}}$ & 0.0874 & 0.1763 & 0.3639 & 0.5773 & 0.8931 & 1.1917 & 1.7320 & 2.7474 & 5.6712 & 11.4300 \\
$\beta_{\mathrm{EST}}$ & 0.0819 & 0.1518 & 0.373 & 0.5779 & 0.8351 & 1.175 & 1.7265 & - & - & - \\
$R E(\beta)=\Delta \beta / \beta$ & 0.0629 & 0.1389 & 0.0250 & 0.0010 & 0.0649 & 0.0140 & 0.0031 & & & \\
$R L H R(\%)$ & 93.71 & 86.10 & 102.50 & 100.10 & 93.51 & 98.60 & 99.68 & - & - & - \\
$R M S E$ & 0.0055 & 0.0177 & 0.0154 & 0.0133 & 0.0285 & 0.0269 & 0.0250 & & & \\
\hline
\end{tabular}

Table 3. Initial $R L H R$ of the RHGC algorithm extended by erosion with step $O_{\mathrm{ALL}} \leq 100$ objects

\begin{tabular}{ccccccccccc}
\hline Text Angle & $5^{\circ}$ & $10^{\circ}$ & $20^{\circ}$ & $30^{\circ}$ & $40^{\circ}$ & $50^{\circ}$ & $60^{\circ}$ & $70^{\circ}$ & $80^{\circ}$ & $85^{\circ}$ \\
\hline$\beta_{\mathrm{REF}}$ & 0.0874 & 0.1763 & 0.3639 & 0.5773 & 0.8931 & 1.1917 & 1.7320 & 2.7474 & 5.6712 & 11.4300 \\
$\beta_{\mathrm{EST}}$ & 0.0819 & 0.1552 & 0.3455 & 0.5625 & 0.8351 & 1.175 & 1.7265 & 2.5221 & 4.8169 & - \\
$\operatorname{RE}(\beta)=\Delta \beta / \beta$ & 0.0629 & 0.1196 & 0.0505 & 0.0256 & 0.0649 & 0.0140 & 0.0031 & 0.0820 & 0.1506 & \\
$\operatorname{RLHR}(\%)$ & 93.71 & 88.03 & 94.94 & 97.44 & 93.51 & 98.60 & 99.68 & 91.80 & 84.94 & - \\
$\operatorname{RMSE}$ & 0.0055 & 0.0154 & 0.0164 & 0.0160 & 0.0296 & 0.0279 & 0.0259 & 0.0832 & 0.2953 & \\
\hline
\end{tabular}

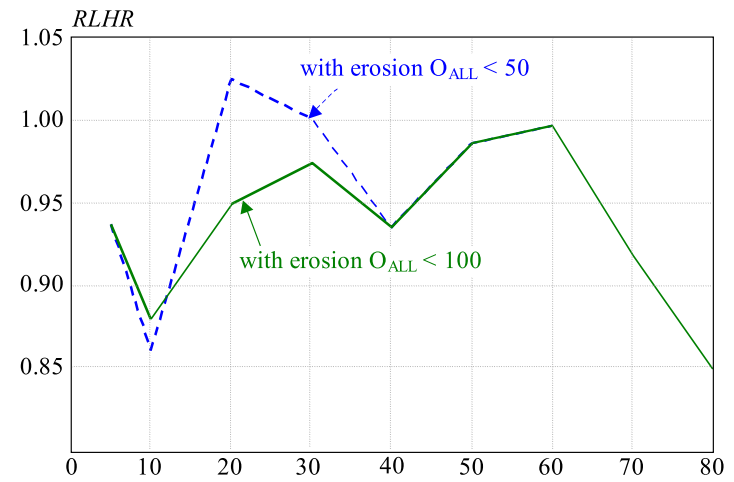

Fig. 7. Initial $R L H R$ of the RHGC algorithm with Rerosion

It is clear, initial skew rate detection using proposed algorithm is accurate in its whole range $\left\{0^{\circ}, \ldots, 90^{\circ}\right\}$, but especially up to $60^{\circ}-70^{\circ}$. Compared to the algorithm [10-11] results are much better in the wider-angle region. If the proposed algorithm is used for final adjustment then text segmentation process is not needed. Due to that, it is clear that proposed method is computationally inexpensive. In addition, it does not depend on column text type. Hence, it is suitable for single or multi column text. However, proposed method can be applied only to printed text.

\section{CONCLUSION}

In the paper, boundary growing method based on rectangular hull gravity center is presented. Method is based on rectangular shape text region expansion. Gravity center of the rectangular object is corner stone for collecting data necessary for reference text line calculation as well

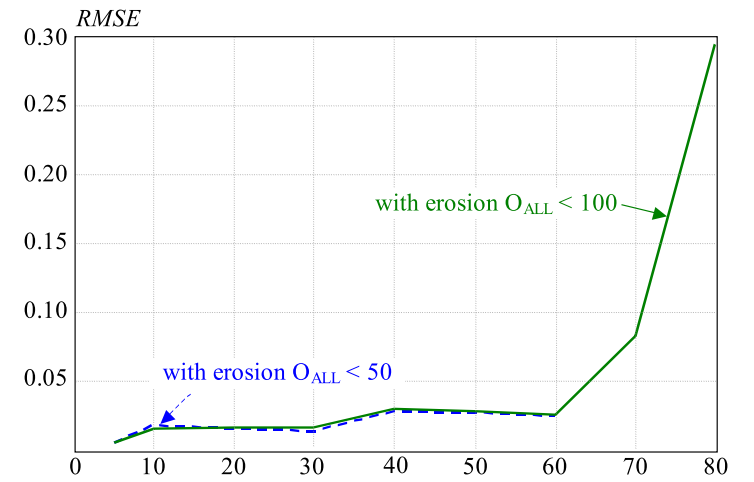

Fig. 8. Aggregate RMSE of RHGC algorithm wiph kerosion

as for initial skew rate estimation. Algorithm experiment gives good $R L H R$ and $R M S E$ results in the whole rotation angle region. Hence, method proves its correctness and usefulness for printed text. In addition, extension of the algorithm with applied erosion is examined. It is suitable for multi line and multi column printed text without segmentation. Obtained results for initial skew rate are promising. In addition, extended method is computationally inexpensive. Due to its correctness method can be integrated partly or completely in the algorithms for handwritten text parameter extraction.

Future work would be oriented toward examining different methods for final adjustments of the skew rate obtained from initial skew rate as well as incorporating it in the algorithms for handwritten text extraction.

\section{REFERENCES}

[1] AMIN, A.-WU, S. : Robust Skew Detection in Mixed Text/ Graphics Documents, Proceedings of 8th ICDAR '05, Seoul, Korea, vol. 1, 2005, pp. 247-251. 
[2] MAnmathA, R.-SRIMAL, N.: Scale Space Technique for Word Segmentation in Handwritten Manuscripts, Proceedings of 2nd International Conference on Scale Space Theories in Computer Vision, 1999, pp. 22-33.

[3] O'GORMAN, L.: The Document Spectrum for Page Layout Analysis, IEEE Transactions on Pattern Analysis and Machine Intelligence 15 No. 11 (1993), 1162-1173.

[4] LOUlOudis, G.-GATOS, B.-PRATIKAKIS, I.-HALATSIS, C.: Text Line Detection in Handwritten Documents, Pattern Recognition 41 No. 12 (2008), 3758-3772.

[5] BASU, S.-CHAUDHURI, C.-KUNDU, M.-NASIPURI, M. -BASU, D. K.: Text Line Extraction from Multi-Skewed Handwritten Documents, Pattern Recognition 40 No. 6 (2007), $1825-1839$.

[6] YAN, H.: Skew Correction of Document Images using Interline Cross-Correlation, CVGIP: Graphical Models and Image Processing 55 No. 6 (1993), 538-543.

[7] WANG, J.-MAZLOR, K. H. L.-SIU, C. H. : Cursive Word Reference Line Detection, Pattern Recognition 30 No. 3 (1997), 503-511.

[8] BRODIĆ, D.-MILIVOJEviĆ, Z.: An Approach to Modification of Water Flow Algorithm for Segmentation and Text Parameters Extraction, Emerging Trends in Technological Innovation (Proceedings of DoCEIS 2010), vol. 314, IFIP AICT, 2010, pp. 324-331.

[9] An-SHATNAWI, A. M.-OMAR, K. : Skew Detection and Correction Technique for Arabic Document Images Based on Centre of Gravity, Journal of Computer Science 5 No. 5 (2009), 363-368.

[10] SHIVAKUMARA, P.-KUMAR, G. H.-GURU, D. S.-NAGABHUSHAN, P.: A Novel Technique for Estimation of Skew in Binary Text Document Images based on Linear Regression Analysis, Sādhanā 30 No. 1 (2005), 69-86.

[11] DHANDRA, B. V.-MALEMATH, V. S.-MILLIKARJUN, H.-HEGADI, R.: Skew Detection in Binary Image Documents Based on Image Dilatation and Region Labeling Approach, Proceedings of 18th ICPR '06, Hong Kong, China, 2006, pp. 954-957.
[12] OTSU, N.: A Threshold Selection Method from Gray-Level Histograms, IEEE Tran. on Systems, Man, and Cybernetics 9 No. 1 (1979), 62-66.

[13] SAUVOlA, L.-PIETIKAINEN, M. : Adaptive Document Image Binarization, Pattern Recognition 33 No. 2 (2000), 225-236.

14] BUKHARI, S. S.-SHAFAIT, F.-BRUESL, T. M. : Adaptive Binarization of Unconstrained Hand-Held Camera-Captured Document Images, Journal of Universal Computer Science 15 No. 18 (2009), 3343-3363.

[15] KHASHMAN, A.-SEKEROGLU, B.: Document Image Binarisation using a Supervised Neural Network, Int. Journal of Neural Systems 18 No. 5 (2008), 405-418.

16] GONZALEZ, R. C.-WOODS, R. E. : Digital Image Procesing, 2nd ed., Prentice-Hall, New Jersey, 2002.

17] PREPARATA, F. P.-ShAmos, M. I.: Computational Geometry: An Introduction, Springer, Berlin, 1985

[18] BRODIĆ, D.-DOKIĆ, B. : Initial Skew Rate Detection Using Rectangular Hull Gravity Center, Proceeding of the 14th International Conference on Electronics (E'2010), Vilnius, Lithuania, 2010.

19] BOLSTAD, W. M.: Introduction to Bayesian Statistics, John Wiley \& Sons, New Jersey, 2004.

[20] BROdić, D.-MILIVOJEVIĆ, D. R.-MILIVOJEVIĆ, Z.: Basic Test Framework for the Evaluation of Text Line Segmentation and Text Parameter Extraction, Sensors 10 No. 5 (2010), $5263-5279$

Received 29 September 2010

Darko Brodić was born in Konjic, BiH, in 1963. He received his BASc, and MASc from Faculty of Electrical Engineering, University of Sarajevo in 1987 and 1990, respectively. Currently, he is with University of Belgrade, Technical Faculty Bor. He is working toward $\mathrm{PhD}$ thesis. His research interests include document image processing. He is the author and coauthor of over 40 journal and conference papers as well as a few books and texbooks.

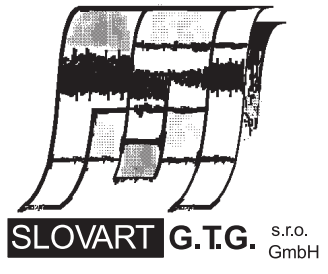

EXPORT - IMPORT
E XPORT - I M P OR T

of periodicals and of non-periodically printed matters, books and CD-ROMs

Krupinská 4 PO BOX 152, 85299 Bratislava 5, Slovakia tel: ++421 263839 472-3, fax: ++421 263839485 info@slovart-gtg.sk; http://www.slovart-gtg.sk

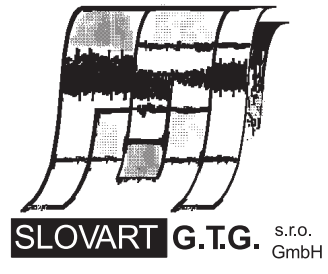

EXPORT - IMPORT 